\title{
Vision based human assistance: Modeling the environment with wearable sensing
}

\author{
Alejandro Rituerto, J. J. Guerrero, Ana C. Murillo \\ ${ }^{I}$ Robotics, Perception and Real Time Group (RoPeRT) \\ Instituto de Investigación en Ingeniería de Aragón (I3A) \\ Universidad de Zaragoza, Mariano Esquillor s/n, 50018, Zaragoza, Spain. \\ Tel.+34-976762707, Fax+34-976762043, e-mail:\{ arituerto, jguerrer, acm\}@ unizar.es
}

\begin{abstract}
El uso de técnicas de visión por computador para aplicaciones de robótica ha crecido en los últimos años. Las cámaras han ganado importancia como sensor debido a la gran cantidad de Información que contiene una imagen, su bajo coste y su pequeño tamaño. Gran parte de la investigación en robótica estudia cómo crear mapas de entornos desconocidos, localizarse y navegar en ellos. El objetivo principal de esta tesis es investigar y adaptar técnicas de visión por computador para la asistencia de personas en la localización y guiado en entornos hechos por el hombre. Más concretamente, este trabajo se centra en el diseño de modelos del entorno para un sistema de mapa jerárquico en los cuales distintas representaciones colaboran a distintos niveles. Los sensores a utilizar serán cámaras tanto convencionales como omnidireccionales. Los sistemas de mapas jerárquicos permiten la utilización conjunta de distintos niveles de detalle y de abstracción para la representación del entorno. En un nivel inferior, un sistema de mapa métrico permitirá una localización precisa con estimación de las distancias reales a objetivo u obstáculos. En un nivel superior, un mapa topológico enriquecido con información semántica, que presentan menor precisión que los métricos, pero permite una mayor abstracción del entorno y la inclusión de información semántica de gran valor a la hora de trabajar con aplicaciones para asistencia de personas. El principal objetivo de esta tesis es contribuir en el campo de la creación de modelos semánticos topológicos para sistemas jerárquicos y asistencia a personas.
\end{abstract}

